High expression of CCL2 in tumor cells and abundant infiltration with CD14 positive macrophages predict early relapse in breast cancer

Heiskala, Marja

2019-01

Heiskala , M , Leidenius , M , Joensuu , K \& Heikkilä , P 2019 , ' High expression of CCL2 in tumor cells and abundant infiltration with CD14 positive macrophages predict early relapse in breast cancer ' , Virchows Archiv , vol. 474 , no. 1, pp. 3-12 . https://doi.org/10.1007/s00428-018-2461-7

http://hdl.handle.net/10138/298729

https://doi.org/10.1007/s00428-018-2461-7

publishedVersion

Downloaded from Helda, University of Helsinki institutional repository.

This is an electronic reprint of the original article.

This reprint may differ from the original in pagination and typographic detail.

Please cite the original version. 


\title{
High expression of CCL2 in tumor cells and abundant infiltration with CD14 positive macrophages predict early relapse in breast cancer
}

\author{
Marja Heiskala $^{1} \cdot$ Marjut Leidenius $^{2} \cdot$ Kristiina Joensuu $^{1} \cdot$ Päivi Heikkilä ${ }^{1}$ \\ Received: 23 May 2018 / Revised: 26 August 2018 / Accepted: 24 September 2018 / Published online: 27 October 2018 \\ (C) Springer-Verlag GmbH Germany, part of Springer Nature 2018
}

\begin{abstract}
Macrophages are important for the function of the innate immune system, and in solid tumors, they represent a significant proportion of the tumor mass. Tumor-associated macrophages (TAM) have a M2 phenotype and show a multitude of pro-tumoral functions, promoting tumor cell survival, proliferation, and dissemination. CCL2, synthesized by tumor and stromal cells, initiates a chemokine cascade inducing these processes. We studied by immunohistochemistry (IHC) the frequency of TAMs and CCL2 expressing cells in three groups of primary tumor (PT)-recurrence (R) pairs, where relapse was recorded within 2 years (group 1), between 5 and 10 years (group 2), and after 10 years (group 3). In our study all established breast cancers were heavily infiltrated by CD68 positive cells. Both in PTs and in R lesions the infiltration was more abundant in the peritumoral than in the intratumoral stroma. The mean frequency of M2 marker and CD14 positive cells in the intratumoral stroma and CCL2 expressing tumor cells was higher in the Rs as compared to the corresponding PTs. In PTs, a high frequency of CD14 positive cells and a high expression of CCL2 by tumor cells was associated with an early recurrence. The findings support the current understanding of immune cell orchestrated development, progression and metastatic spread of breast cancer. Our study showed that a high frequency of CCL2 positive tumor cells and CD14 positive TAMs are significant risk factors for rapid tumor recurrence. Potential targets for intervention are discussed.
\end{abstract}

Keywords Innate immunity $\cdot$ Breast cancer $\cdot$ CCL2 $\cdot$ CD14 positive macrophages $\cdot$ Prognostic markers in breast cancer

\section{Introduction}

Macrophages are an important part of the innate immune system, and they also participate in tissue remodeling. They have a non-redundant role in mammary ductal outgrowth during postnatal mammary gland development [1] via mechanisms similar to those active during breast tumor progression [2].

Kristiina Joensuu

kristiina.joensuu@helsinki.fi; kristiinajoensuu7@gmail.com

Marja Heiskala

maria.heiskala@gmail.com

Marjut Leidenius

marjut.leidenius@hus.fi

1 Department of Pathology and HUSLAB, Helsinki University Hospital and University of Helsinki, FIN-00290 Helsinki, Finland

2 Breast Surgery Unit, Comprehensive Cancer Center Helsinki University Hospital, P.O Box 163, 00290 HUS, Helsinki, Finland University of Helsinki, Central Hospital,

FIN-00290 Helsinki, Finland
Macrophages, with CD68 as the common marker of the cell lineage, represent a significant proportion of the tumor mass in solid malignancies. They originate from blood monocytes, which differentiate into two schematically identified categories, M1 (classically activated) and M2 (alternatively activated) [3]. Tumor-associated macrophages (TAM) have the tumor promoting M2 phenotype [4], and this population can further be divided into M2a, M2b, M2c, and M2d subcategories with different functions [5]. M2c-macrophages, with CD163 as validated marker, represent the immunosupressive end of the continuum. Human monocytes and macrophages also widely express CD14 during migration and differentiation towards CD14 + CD16 ++ non-classical tumor promoting monocytes [6]. M1 polarized anti-tumor functions harboring macrophages over express co-stimulatory molecules, such as CD80 [7-9]. The recruitment of TAM and their interaction with metastasizing tumor cells is dependent on tumoral and stromal-derived CCL2, pivotal for the chemokine cascade that initiates metastatic seeding of breast cancer cells [10-15].

Previously, a high frequency of CD68 positive macrophages has been shown to associate with higher tumor grade, 
estrogen receptor (ER) and progesterone receptor (PR) negativity, human epithelial growth factor receptor 2 (HER-2) positivity, and a basal phenotype [16]. The number of CD163 positive cells in breast tumor samples has been shown to correlate with fast proliferation, poor differentiation, and ER negativity [17]. The overall frequency of CD68 or CD163 positive cells in tumor samples has, however, not been shown to have an independent prognostic significance [18, 19].

In the current study, we examined the frequency and localization of the CD68, CD14, CD206, CD163, and CD80 positive macrophages in primary breast tumors and the corresponding recurrences, in order to monitor differences between primary and recurrent tumors, and between early versus late recurring tumors. We also investigated the expression of CCL2 to elucidate its role in primary and recurrent tumor development. Finally, the correlation of the expression of these markers with the traditional clinicopathological parameters was investigated.

\section{Materials and methods}

\section{Patients and tissue samples}

Paraffin-embedded tissue blocks from primary tumors (PTs) of 137 patients and their corresponding recurrent $(\mathrm{R})$ lesions were collected from the archives of the Department of Pathology, Helsinki University Hospital as described previously [20]. The primary breast cancer surgery had been conducted in 1974-2006. The cases were selected depending on the time lapse from the primary operation to the first recurrence to represent quick ( $\leq 2$ years), intermediate (5-10 years), and slow ( $>10$ years) progressors. All consecutive cases matching with the required primary treatment-relapse interval were recruited. They were divided into three groups: Group 1 $n=41$, tumors with $\mathrm{R}$ within 2 years after primary surgery, group $2 n=57$, with $\mathrm{R}$ after 5-10 years, and group $3 n=39$ with $\mathrm{R}$ after $>10$ years (range 10 to 23 years). The archival slides were re-examined, and the histological tumor type and grade was assigned based on the criteria of Elston and Ellis [20]. The clinicopathologic characteristics of the patients and their cancers are summarized in Table 1. The Ethical Committee of the Helsinki University Central Hospital approved the study protocol.

\section{Immunohistochemistry}

The expression of CD68 and CD14, pan-monocyte/macrophage markers, CD163 and CD206, M2 macrophage markers, CD80, an M1 macrophage marker, and CCL2, a pivotal chemokine-associated with macrophage recruiting and maturation, in primary breast cancer and corresponding recurrences was investigated. The material consisted of early,
Table 1 Clinicopathologic parameters of the 137 breast cancer patients and the site of recurrence

Group $1 n=41$ Group $2 n=57$ Group $3 n=39$

\begin{tabular}{|c|c|c|c|}
\hline \multicolumn{4}{|c|}{ Age at surgery of primary tumor (PT) } \\
\hline$<50$ years & 19 & 20 & 18 \\
\hline$\geq 50$ years & 22 & 37 & 21 \\
\hline \multicolumn{4}{|l|}{ Tumor size } \\
\hline$\geq 20 \mathrm{~mm}$ & 14 & 28 & 24 \\
\hline$<20 \mathrm{~mm}$ & 26 & 28 & 15 \\
\hline system missing & 1 & 1 & \\
\hline \multicolumn{4}{|l|}{ Lymph node } \\
\hline Negative & 14 & 34 & 21 \\
\hline Positive & 24 & 20 & 13 \\
\hline System missing & 3 & 3 & 5 \\
\hline \multicolumn{4}{|l|}{ Grade } \\
\hline 1 & 4 & 7 & 8 \\
\hline 2 & 22 & 35 & 26 \\
\hline 3 & 15 & 15 & 5 \\
\hline \multicolumn{4}{|l|}{ Histological type } \\
\hline Ductal & 24 & 36 & 16 \\
\hline Lobular & 17 & 19 & 23 \\
\hline Special types & 0 & 2 & 0 \\
\hline \multicolumn{4}{|c|}{ Tissue site of recurrence $(\mathrm{R})$} \\
\hline Skin & 6 & 10 & 11 \\
\hline Soft tissue & 6 & 12 & 5 \\
\hline Subcutaneous tissue & 12 & 16 & 15 \\
\hline Lung & 0 & 4 & 2 \\
\hline Brain & 2 & 2 & 0 \\
\hline Lymph node & 2 & 1 & 2 \\
\hline Ovary & 0 & 1 & 0 \\
\hline Bone & 3 & 6 & 4 \\
\hline Liver & 5 & 2 & 0 \\
\hline Pleura & 0 & 1 & 0 \\
\hline Peritoneum & 2 & 1 & 0 \\
\hline Mesenterium & 1 & 0 & 1 \\
\hline Larynx & 1 & 0 & 0 \\
\hline Uterus & 1 & 0 & 1 \\
\hline Duodenum & 0 & 1 & 0 \\
\hline
\end{tabular}

Note: In group 1, recurrences were detected within 2 years, in group, 2 from 5 to 10 years, and in group 3,> 10 years after primary surgery. $R$ was defined as any local or regional recurrence or any distant metastatic disease

intermediate, and late recurring tumors. The grading of the level of the expression of the selected marker proteins was based on viewing the entire tumor sections at the $\times 20$ magnification, and specifying the type of the marker positive cells using $\times 40$ magnification. The method gives a crude estimate of the frequency of positive cells, not allowing a precise quantification. However, being able to evaluate a large number (137) of primary tumor/recidive pairs allows confidence on 
the significance of the detected differences in the expression of the markers. Moreover, the repeatability of the grading, tested by re-evaluating an aliquot of samples, was $100 \%$. Inter-individual variation on evaluating the immunohistochemical result of the macrophage phenotype-related antigenes, CD68, CD14, CD80, CD163, and CD206 and the macrophage chemo attractant MCP-1/CCL2was negligible, due to the clarity of the cutting points agreed upon: 0 , no antigen expression; + 1, 5-29\% of antigen expressing cells; $+2,30-69 \%$ of antigen expressing cells; and $+3,70-100 \%$ of antigen expressing cells.

For staining result for ER, PR, HER2, and MIB1 the percentage of positive stained tumor cells (range $0-100 \%$ ) was recorded according to a standard protocol [20]. All tumors with an over expression of HER2 at protein level (see ref. 20) were tested for HER2 gene amplification by Inform HER2 Dual ISH test (inform HER2 dual in situ hybridization).

\section{Immunohistochemical methods and chemicals}

Four-m thick sections were deparaffinized in xylene and rehydrated. Antigen retrieval was done by microwaving in $10 \mathrm{mM}$ citric acid monohydrate for $1 \times 5 \mathrm{~min}$ at $900 \mathrm{~W}$ and for $3 \times 5 \mathrm{~min}$ at $600 \mathrm{~W}$. Endogenous peroxidase activity was blocked by treatment with $0.5 \% \mathrm{H} 2 \mathrm{O} 2$.

The slides were incubated overnight in a refrigerator at $+4{ }^{\circ} \mathrm{C}$ with appropriate dilutions of the primary antibodies. The same procedure was used for negative controls, omitting the primary antibody. The reaction was visualized by the Elite ABC Kit (Vectastain, Vector Laboratories, Burlingame, CA, USA). As primary antibodies the following reagents were used: mouse monoclonal clones anti-CD68 PG-M1, dilution 1:200 (DacoCytomation Denmark), anti-CD14 clone 7, dilution 1:50 (Labb Vision Corporation, USA), anti-CD163 clone 10D6, dilution 1:200 (Leica Biosystems Newcastle Ltd, UK), anti-CD206 clone 0387, dilution 1:2000 (Atlas Antibodies $\mathrm{AB}$, Sweden), anti-MCP-1/CCL2 clone $2 \mathrm{~dB}$, dilution 1:5000 (LifeSpan BioSciences, Inc. USA), anti-ERalfa clone 6F11, dilution 1:50 (Novo Castra Newcastle, UK), anti-PR alfa clone 636, dilution 1:100 (DacoCytomation, Denmark), anti-HER2 clone CB11, dilution 1:400 (Novo Castra, UK), anti-Ki67 clone MIB-1, dilution 1:75 (Daco Cytomation, Denmark), and a rabbit polyclonal antibody anti-CD80 CD/ 80/B71, dilution (Sino Biological Inc., P.R. China).

For staining result for ER, PR, HER2, and MIB1, the percentage of positive stained tumor cells (range $0-100 \%$ ) was analyzed [20]. All tumors with over expression of HER2 at protein level $(2+$ and $3+$; see ref. 20$)$ were tested for HER2 gene amplification by Inform HER2 Dual ISH test (inform HER2 dual in situ hybridization). The HER2 gene was targeted by a dinitrophenyl labeled probe (INFORM HER2 Dual ISH DNA Probe Cocktail, 780-4422, Roche/Ventana/ Tuscon, AZ, USA 780-4422) and visualized with
VENTANA ultraView Silver ISH DNP (SISH) Detection (760-098, Roche/Ventana/Tuscon, AZ, USA). The chromosome 17 centromere was localized with a digoxigenin labeled probe (INFORM HER2 Dual ISH DNA Probe Cocktail (7804422, Roche/Ventana/Tuscon, AZ, USA) and detected using VENTANA ultraView Red ISH DIG Detection (780-4422, Roche/Ventana/Tuscon, AZ, USA). The HER2 signals were seen as black signals and Chr17 as red signals under light microscope.

Normal human tonsil and placenta tissue were used as positive controls for macrophage and CCL2 markers, and normal and malignant breast tissue for the breast epithelium-related markers. We evaluated the entire tumor area from one representative section from the PT and the R. The results were scored independently by three pathologists (KJ, MH, PH).

\section{Statistical methods}

All statistical analyses were performed using SPSS 24.0 for Windows (SPSS Incorporation, Chicago, IL, USA). The differences between the expression of the markers in PTs and the corresponding Rs within the groups were tested using the paired samples $t$ test. Kruskal-Wallis test and the MannWhitney $U$ test was used for comparing differences between the groups. For analyzing the association of the expression of the markers with the clinicopathologic parameters, ER, PR, Ki67, and HER2, we used the categorical two-tailed Pearson's chi-square test. For Pearson's chi-square tests the cutoff point for negativity versus positivity was $<+2$ versus $\geq+2$ for CD68, CD80, CD163, CD206, and CCL2. For ER and PR the cutoff point for positivity was $1 \%$, and for Ki67 $\geq 14 \%$. For HER 2 only, those tumors with positive gene amplification were considered positive.

Probability values $p<0.05$ were considered significant except in the Mann-Whitney $U$ test, where $P<0.0167(<0.5 / 3)$ was used.

\section{Results}

\section{CD68}

In the entire tumor set, the frequency of intratumoral CD68 positive leukocytes was high $(\geq 2+)$ in 46 cases $(33.6 \%)$ of PTs and in 43 cases $(31.4 \%)$ of Rs. The peritumoral stromal frequency of CD68 positive leukocytes was high $(\geq 2+)$ in 76 cases $(55.5 \%)$ of PTs and in 73 cases $(53.3 \%)$ of Rs. The mean frequency of intratumoral or peritumoral CD68 positive leukocytes did not differ between the PTs and the Rs ( $P=0.433$, $P=0.927$, Figs. 1-3). The mean frequency of intratumoral versus peritumoral CD68 positive leukocytes differed significantly: the CD68 positive cells were more frequent in the peritumoral than in intratumoral stroma, $P=0.0001$ (data not 
Fig. 1 Differences in the mean frequency of positive cells $(0,1+$, $2+, 3+)$ between the PTs and their corresponding Rs. *Significant difference. Paired samples $t$ test was used

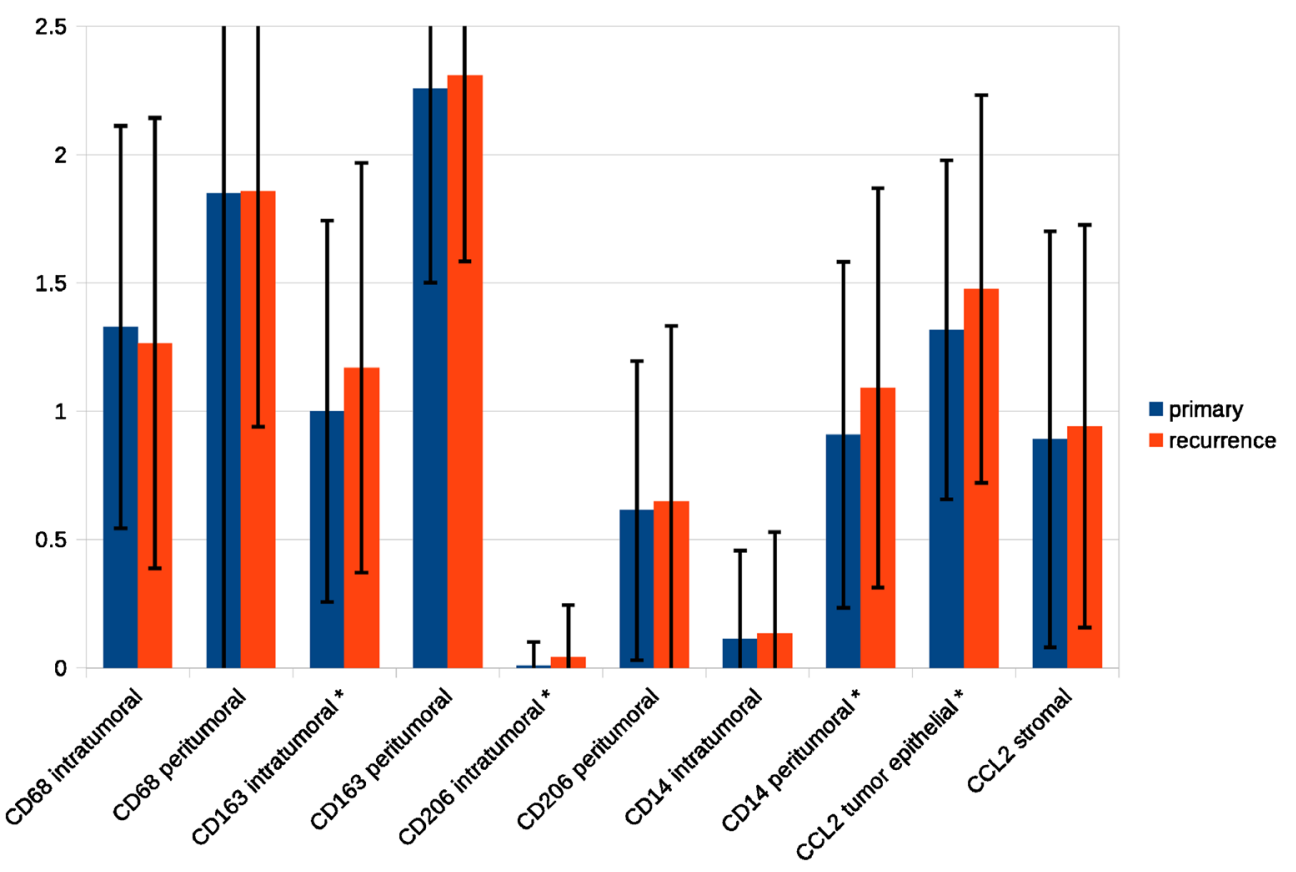

shown). The early relapsing group 1 and the later recurring groups 2 and 3 did not differ from each other with respect to the frequency on CD68 positive cells.

The frequency of peritumoral CD68 expressing leukocytes in PTs correlated positively with ER $(P=0.007)$ and PR $(P=$ 0.017 , Table 2). Both the intratumoral and peritumoral frequency of CD68 expressing leukocytes correlated positively with MIB1 positivity ( $\geq 14 \%$ as cutoff of positive tumor cells), $(P=0.016$ in both, Table 2$)$.

The frequency of CD68 positive leukocytes did not correlate with the other clinicopathological parameters: patient age, axillary lymph node status, tumor size, tumor grade, histological subtype, nor with the HER2 amplification status.

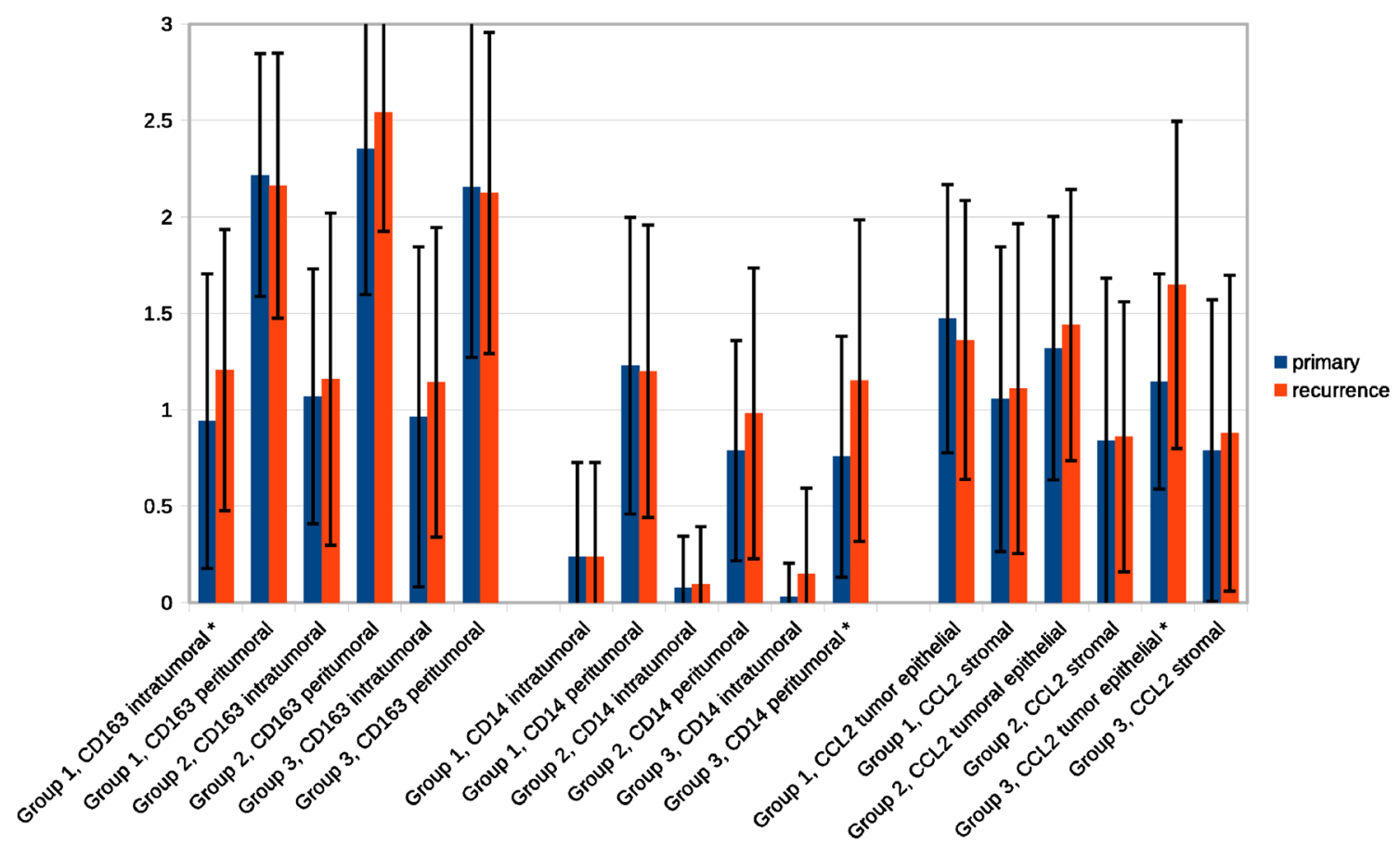

Fig. 2 Differences in the mean frequency of tumor epithelial CCL2, intratumoral, and peritumoral stromal CD163 and CD14 positive cells between the PTs and their corresponding Rs in groups 1, 2, and 3.
*Significant difference. Group 1 represents tumors with Rs within 2 years. In group 2, Rs were detected at 5 to 10 years, and in group $3>$ 10 years after primary surgery. Paired samples $t$ test was used 
Fig. 3 IHC showing the expression of DC68, CD14, CD163, CD206, and CCL2 in $\mathrm{PTs}$ and corresponding residives ( $R>5$ years, $R>10$ years)

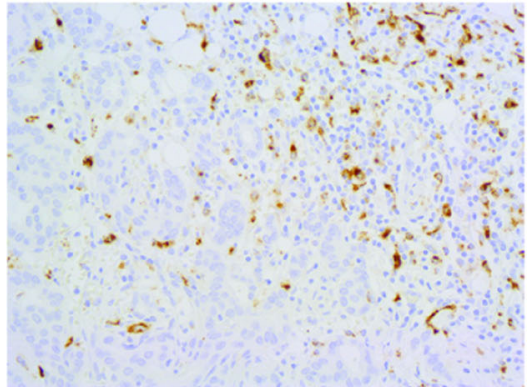

\section{68 in PT}

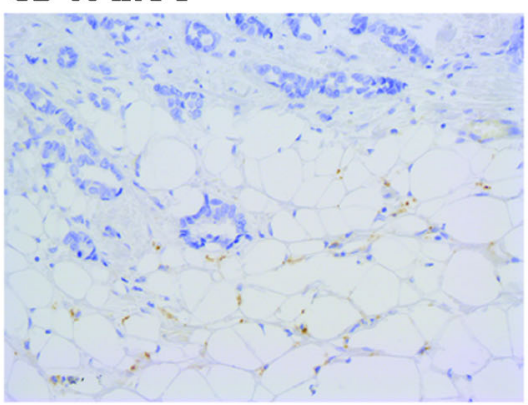

CD 14 in PT

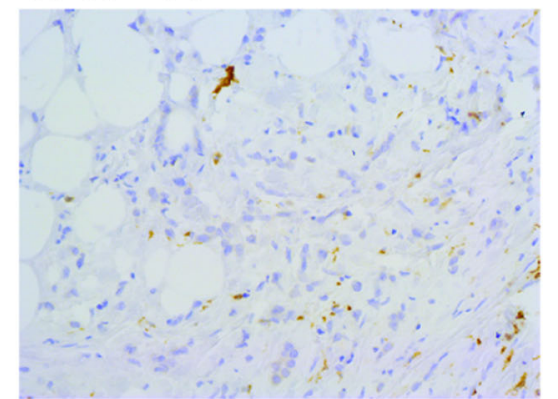

\section{163 in PT}

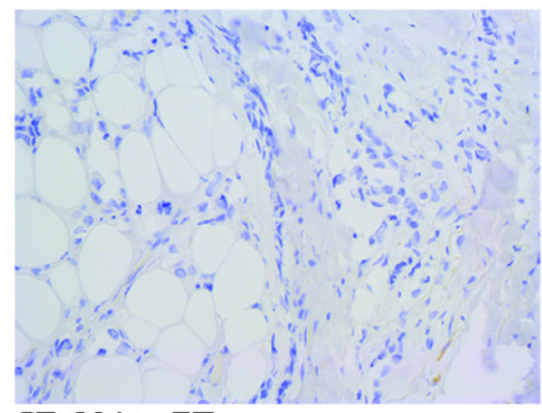

\section{206 in PT}

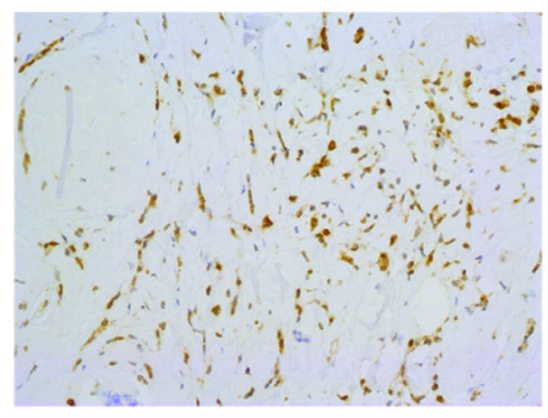

CCL2 in PT

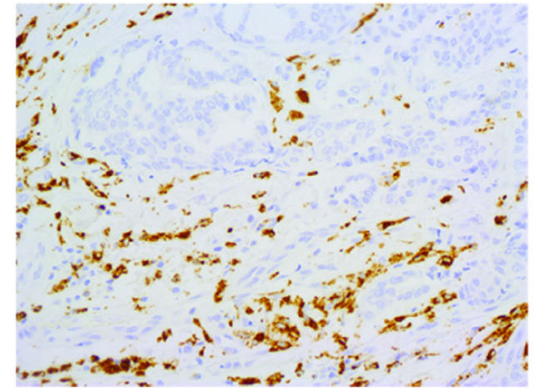

CD 68 in $R>5$ years

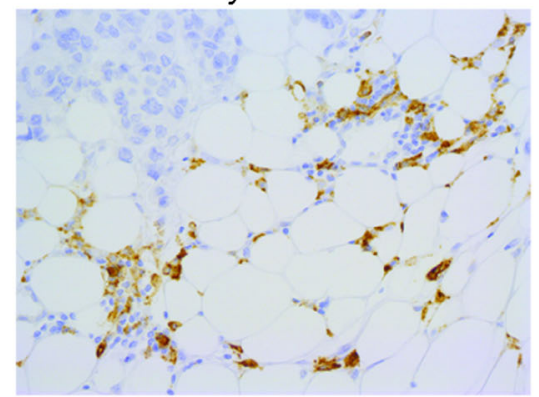

CD 14 in $R>10$ years

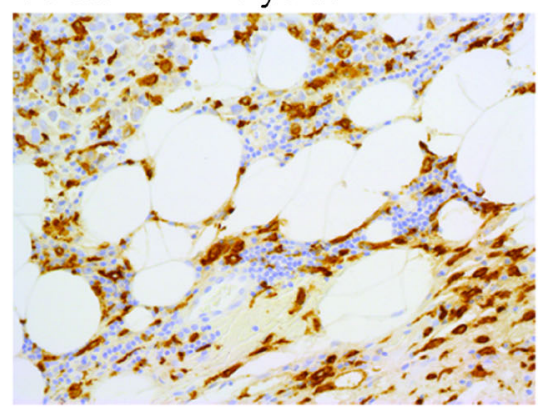

CD 163 in $R>10$ years

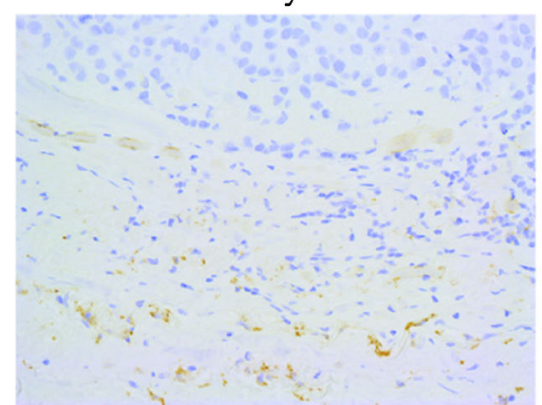

CD 206 in $R>10$ years

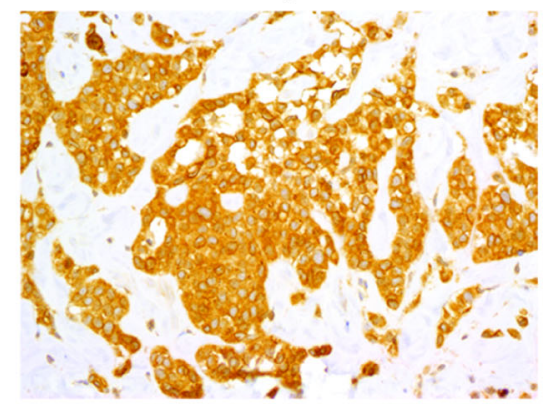

CCL 2 in $R>10$ years 
Table 2 Relationship between CD68, CD163, CD206, CCL2, CD14, ER, PR, and Ki67 IHC expression, and HER2 gene amplification in PTs of 137 breast cancer patients was investigated and the significant results are shown

\begin{tabular}{|c|c|c|c|}
\hline & $\begin{array}{l}\text { Number of cases with low frequency } \\
\text { of intratumoral CD68 positive cells }\end{array}$ & $\begin{array}{l}\text { Number of cases with high frequency } \\
\text { of intratumoral CD } 68 \text { positive cells }\end{array}$ & $P$ value \\
\hline \multicolumn{4}{|l|}{ CD68 } \\
\hline MIB $1<14 \%$ & $44(71.0 \%)$ & $18(29.0 \%)$ & \\
\hline \multirow[t]{2}{*}{$\mathrm{MIB} 1 \geq 14 \%$} & $26(49.1 \%)$ & $27(50.9 \%)$ & 0.016 \\
\hline & $\begin{array}{l}\text { Number of cases with low frequency } \\
\text { of peritumoral CD68 positive cells }\end{array}$ & $\begin{array}{l}\text { Number of cases with high frequency } \\
\text { of peritumoral CD68 positive cells }\end{array}$ & \\
\hline ER negative & $24(55.8 \%)$ & $19(44.2 \%)$ & \\
\hline ER positive & $25(30.9 \%)$ & $56(69.1 \%)$ & 0.007 \\
\hline PR negative & $21(55.3 \%)$ & $17(44.7 \%)$ & \\
\hline PR positive & $28(32.6 \%)$ & $58(67.4 \%)$ & 0.017 \\
\hline MIB1 < $14 \%$ & $34(49.3 \%)$ & $35(50.7 \%)$ & \\
\hline $\mathrm{MIB} 1 \geq 14 \%$ & $15(27.8 \%)$ & $39(72.2 \%)$ & 0.016 \\
\hline \multicolumn{4}{|l|}{ CD163 } \\
\hline & $\begin{array}{l}\text { Number of cases with low frequency } \\
\text { of intratumoral CD163 positive cells }\end{array}$ & $\begin{array}{l}\text { Number of cases with high frequency } \\
\text { of intratumoral CD163 positive cells }\end{array}$ & $P$ value \\
\hline HER2ISH negative & $68(73.9 \%)$ & $24(26.1 \%)$ & \\
\hline \multirow[t]{2}{*}{ HER2ISH positive } & $14(100.0 \%)$ & $0(0.0 \%)$ & 0.030 \\
\hline & $\begin{array}{l}\text { Number of cases with low frequency } \\
\text { of peritumoral CD163 positive cells }\end{array}$ & $\begin{array}{l}\text { Number of cases with high frequency } \\
\text { of peritumoral CD163 positive cells }\end{array}$ & \\
\hline PR negative & $11(28.9 \%)$ & $27(71.1 \%)$ & \\
\hline PR positive & $8(9.8 \%)$ & $74(90.2 \%)$ & 0.007 \\
\hline MIB1 < 14\% & $16(24.6 \%)$ & $49(75.4 \%)$ & \\
\hline $\mathrm{MIB} 1 \geq 14 \%$ & $3(5.6 \%)$ & $51(94.4 \%)$ & 0.005 \\
\hline \multicolumn{4}{|l|}{ CD14 } \\
\hline & $\begin{array}{l}\text { Number of cases with low frequency } \\
\text { of peritumoral CD14 positive cells }\end{array}$ & $\begin{array}{l}\text { Number of cases with high frequency } \\
\text { of peritumoral CD14 positive cells }\end{array}$ & $P$ value \\
\hline $\mathrm{MIB} 1<14 \%$ & $58(95.1 \%)$ & $3(4.9 \%)$ & \\
\hline $\mathrm{MIB} 1 \geq 14 \%$ & $38(74.5 \%)$ & $13(25.5 \%)$ & 0.002 \\
\hline
\end{tabular}

\section{CD163}

The frequency of intratumoral CD163 positive leukocytes in PTs was high $(\geq 2+)$ in $25(18.2 \%)$ cases and in $34(24.8 \%)$ cases in Rs. The frequency of peritumoral CD163 positive leukocytes was high in $103(75.2 \%)$ cases in PTs and in 106 (77.4\%) cases in Rs.

The mean frequency of intratumoral CD163 positive cells was significantly higher in the Rs than in their corresponding PTs $(P=0.031$, Fig. $1-3)$. The most significant difference was seen in Group $1(P=0.048$, Fig. 2). The frequency of the peritumoral CD163 positive cells did not significantly differ between the PTs and the Rs, $(P=0.499$, Fig. 1).

In the Rs, the frequency of the peritumoral $\mathrm{CD} 163$ positive leukocytes differed between the three groups $(P=0.031$, Kruskal-Wallis test, data not shown). The frequency of CD163 positive cells was significantly higher in group 2 as compared to group 1 ( $P=0.013$, Mann-Whitney $U$ test, data not shown), but in group 3, the frequency was again at the same level as in group 1.
The frequency of peritumoral CD163 positive cells was significantly higher than that of the intratumoral stroma in both PTs and the Rs, $(P=0.0001)$, data not shown.

The frequency of peritumoral CD163 positive cells in PTs correlated positively with MIB1 $(P=0.005)$ and PR $(p=$ 0.007), Table 2. There was no correlation between CD163 and ER, the prognostic clinicopathological parameters, nor HER2 amplification.

\section{CD14}

The frequency of intratumoral CD14 positive cells was high $(\geq 2+)$ in $1(0.7 \%)$ PT and in $2(1.5 \%)$ Rs. The frequency of peritumoral CD14 positive myelo-monocytes was high in 12 (14.6\%) PTs and in 39 (28.5\%) Rs. The mean frequency of intratumoral and peritumoral CD14 positive cells was higher in the $\mathrm{R}$ tumors than in their primary counterparts (Fig. 1). This difference was significant with regard to the peritumoral cells $(P=0.029$, Fig. 1$)$. When analyzing the expression of CD 14 in the groups 1,2, and 3, the expression was noted to 
be significantly higher in the peritumoral stroma of the Rs as compared to PTs in group $3(P=0.017$, Fig. $1-3)$. Both intratumoral and peritumoral frequency of $\mathrm{CD} 14$ positive cells in PTs was the higher the sooner the recurrence happened ( $P=0,031, P=0,007$, Kruskal-Wallis test, data not shown). In the peritumoral stroma, the difference between groups 1 and 2 , and between groups 1 and 3 was significant $(P=$ $0.006, P=0.016$, Mann-Whitney $U$ test, data not shown). The frequency of neither intratumoral nor peritumoral CD14 positive leukocytes correlated with the prognostic clinicopathological parameters.

\section{CD206}

The frequency of intratumoral CD206 positive cells was high in none of the PTs nor in the Rs. In primary tumors, there were three cases $(3.6 \%)$ and in recurrences $5(3.6 \%)$ cases with a low frequency (1+) of intratumoral CD206 expression. The frequency of peritumoral CD206 positive cells was high in 4 (2.9\%) cases in PTs and in 7 (7.3\%) Rs.

The mean frequency of intratumoral CD206 positive leukocytes was significantly higher in the recurrences as compared to the PTs $(P=0.045$, Fig. $1-3)$. The peritumoral positivity did not differ between the PTs and the Rs. The frequency of peritumoral CD206 positive cells was significantly higher than the intratumoral both in PTs and in Rs $(P=0.0001)$, data not shown.

There were no significant differences between the groups in intratumoral nor in peritumoral CD206 positivity.

CD206 positivity did not correlate with the prognostic clinicopathological parameters (data not shown).

\section{CD80}

There was only a few cases with low frequency of CD80 positive leukocytes in the whole tumor set, and no statistical analyses were performed.

\section{CCL2/MCP-1}

The expression of CCL2 in malignant epithelial cells in PTs was high $(\geq 2+)$ in 42 cases $(30.7 \%)$ and the frequency of stromal CCL2 positive cells in PTs was high in 25 cases $(18.2 \%)$. In Rs, CCL2 expression in tumor cells was high in $54(39.4 \%)$, and in stromal cells in $31(22.6 \%)$.

The mean CCL2 expression in tumor epithelial cells was significantly higher in the Rs compared to the corresponding PTs ( $P=0.024$, Figs. 1 and 2$)$. There was no significant difference in the frequency of CCL2 positive stromal cells between the PTs and their Rs ( $P=0.494$, Fig. 1), whereas the mean tumor epithelial cell expression of CCL2 was significantly higher in the Rs than in the PTs in group $3(P=0.0001$, Figs. 2 and 3).
The tumor epithelial cell CCL2 positivity was lower in the PTs in the late relapsing group 3 as compared to the earlier relapsing groups 1 and 2, although the difference did not reach statistical significance. The tumor epithelial cell CCL2 positivity was the higher the earlier the recurrent lesion was diagnosed (Fig. 2). CCL2 positivity did not correlate with the prognostic clinicopathological parameters (data not shown).

\section{Discussion}

We screened the expression on CD68 and CD14, pan-monocyte/macrophage markers, CD163 and CD206, M2 macrophage markers, CD80, an M1 macrophage marker, and CCL2, a pivotal chemokine associated with macrophage recruiting and maturation, in primary breast cancer (primary tumor, PT) and corresponding recurrences (R). The material consisted of early, intermediate, and late recurring tumors. The goal was to evaluate the impact of macrophage infiltration and M2 maturation on the biological behavior of the tumor, and to detect potential targets for intervention in patients with breast cancer.

Pan-macrophage marker positive cells were abundant in all established tumors, and the infiltration was heavier in the tumor surrounding stroma than in the intratumoral stroma. M2 marker and CD14 positive cells were more frequent in Rs than in the corresponding PTs. CCL2, the major recruiter of myeloid-derived macrophages, was also expressed at a higher rate in Rs than in PTs. Both CCL2 and CD14 showed a tendency of a relatively high expression in those PTs which were associated with an early recurrence.

It was shown that the CD68 positive cells were more frequent in the peritumoral than in intratumoral stroma. Neither the PTs and R lesions, nor the different prognostic groups differed from each other with respect to the expression of CD68. All established tumors were heavily infiltrated with CD68 positive cells, which is in accordance with previous findings. Overall CD68 expression has previously been associated with non-favorable features of the tumor, such as ER negativity and HER2 positivity [16]. In our study the peritumoral CD68 positivity correlated with ER, suggesting that the prognostic significance of CD68 positive cells depends on the localization: in non-hypoxic regions macrophages tend to maturate towards M1 phenotype, and may in breast cancer associate with a favorable prognosis. However, both peritumoral and intratumoral CD68 expression correlated positively with MIB1 status, which is in accordance with previous findings suggesting an association of $\mathrm{CD} 68$ positive cell infiltration with tumor aggressivity. In our study, DC68 infiltration did not, however, have prognostic significance as measured by the time of recurrence.

The intratumoral but not the peritumoral frequency of CD163 positive cells was higher in Rs than in PTs, supporting 
the previous finding that M2 macrophages play an important role in tumor spread and associate with tumors with features of poor prognosis [17]. In the Rs, the frequency of the peritumoral CD163 positive leukocytes seemed to peak in group 2 (Fig.2), but with lack of further correlation with the time of recurrence the significance of this finding remains uncertain.

Only few tumors in our study were positive for CD206, suggesting that the majority of the M2 macrophages were of migratory type, which do not express CD206 [21]. The mean frequency of intratumoral CD206 positive leukocytes was significantly higher in the Rs compared to the Pts, which is in accordance with the concept of macrophage M2 type maturation in tumor microenvironment. Due to the sparse expression, the impact of the finding remains elusive.

CD80 was expressed in only few tumor samples, which is in accordance with the understanding that tumor inflitrating macrophages quite uniformly represent the M2 polarization.

CD14 was more abundantly expressed in the peritumoral than in the intratumoral stroma. CD14 positive cells were more abundant in both peritumoral and intratumoral stroma in those PTs, which were associated with an early R, than in those, which recurred later. The expression was always higher in the R than in the corresponding PT. The difference in the peritumoral infiltration of CD14 positive cells between PT and $\mathrm{R}$ was significant in group 3, where the frequency of CD14 positive cells was relatively low in PTs. In inflammation monocytes invade tissue by interaction of complementary pair of CCR2/CCL2 or/and CCR5/CCL5, and the resulting CD14 + monocyte count increase is a hallmark of human inflammatory disease [22]. CD14 positive TAM population represents a newly recruited subset of monocytes, and the tumor microenvironment directs the newly recruited CCR2 over expressing CD14++CD16- cells to adopt the tumor promoting intermediate or non-classical phenotype. CD14 ++ CD16- monocytes are characterized by an enhanced expression of CCR2, the cell surface receptor of CCL2 [13]. The frequency of CD14 positive cells had the same kinetics as the level of CCL2 expression in tumor cells in our study, suggesting that the CCL2-pathway effectively recruits CD14 positive monocytes/macrophages, and both CCL2 and CD14 can be seen as markers of potential tumor spread.

The mean tumor epithelial cell CCL2 expression was significantly higher in the Rs compared to the corresponding PTs. In group 3 (recurrence after 10 years), the increase of the number of CCL2 positive tumor cells in the R lesions as compared to the corresponding PTs reached statistical significance. The mean tumor epithelial cell expression of CCL2 in the PTs was the higher the earlier the R lesion was diagnosed. This finding is in accordance with the earlier studies, where an increased expression of CCL2 in cancer microenvironment has been shown to be required for tumor progression and metastasis [23-25]. It has been shown in an experimental setting, too, that CCL2 together with IL-6 supports the maturation of M2-type macrophages, characterized by the expression of the plasma membrane receptor CD14 and an increased expression of mannose receptor CD206 [25], further underlining the importance of CCL2 in tumor progression.

The co-expression of CCL2 and CD14 may turn out to be independent prognostic indicators in breast cancer. CCL2 is also a potential target for intervention.

CCL2 positivity did not correlate with ER, PR, MIB1, and HER2 amplification nor the other prognostic clinicopathological parameters.

The MIB-1 proliferation index has been shown to correlate directly with tumor size, nodal status, overexpression of $\mathrm{p} 53$ and Neu, and the DNA index; and inversely with estrogen and progesterone receptor status [26]. A low proliferation index correlates with a longer relapse-free interval and overall survival. In our study the frequency of peritumoral and intratumoral CD68 expressing leukocytes and that of peritumoral CD163 positive cells correlated directly with MIB-1 expression. This is in accordance with the assumption that the infiltration of TAM predicts a poor prognosis, although significant correlation was not reached in this study.

Over expression of $\mathrm{ER} \alpha$ is a well-established prognostic factor in breast cancer patients. ER $\alpha$-positive breast cancers are associated with a slower tumor growth and a better overall prognosis. However, the value of ER $\alpha$ status as an independent prognostic variable has been diluted by its association with other prognostic factors and treatment modalities. ER status may be more important for prognosis in node-negative cases than in node-positive [27]. In this study, the frequency of peritumoral but not intratumoral CD68 expressing leukocytes in PTs correlated positively with ER expression, suggesting that macrophages specifically in normo-oxic areas may associate with tumors with a less aggressive biological behavior. ER status did not correlate with the other macrophage markers nor CCL2.

The event that initiates the cascade of M2 macrophage recruitment and maturation is the constitutive IL6-STAT3 pathway activation in cancer cells, and the secretion of CCL2. Inhibition of myeloid macrophage recruitment and maturation towards tumor promoting alternatively activated M2 macrophages is a potential tool in immunologic cancer care.

Inhibition of the production of CCL2 by trabectedin [28], allosteric blocking of the function of CCL2 by "Peptide 3," a dodecapeptide section of CCL2 [29, 30] and suppressing the TAM-derived MMP-9 to reduce tumor cell migration and VEGF activity [31] are examples of successful interventions. A more downstream inhibition of the CCL2/CCR2 pathway by blocking CCL3/CCR1 has also been suggested [32].

In this study, the role of the CCL2 pathway in tumor progression was verified, and the prognostic significance of CCL2 and CD14 in breast cancer was detected. 
Based on our results, we suggest that the coarse needle biopsy necessary in diagnosing breast cancer should be covered by a preventing method due to the risk that it may induce an unfavorable immunologic reaction and facilitate tumor metastatic potential via the CCL2 pathway [33]. The possible benefit of a local intratumoral injection of peptide- or neutralizing antibody-based anti-CCL2 agent at the time of biopsy should be evaluated. Systemic anti-CCL2 treatment as neoadjuvant in patients with breast cancer should also be considered.

Acknowledgements We thank Eija Heiliö for her excellent technical assistance, and Antti Nevanlinna, Msc, for facilitating the statistical analysis.

Author Contributions Marja Heiskala: doing the literature search and planning the study, evaluating the samples, writing the article, and preparing the graphics

Marjut Leidenius: planning the study, writing the article

Kristiina Joensuu: planning the study, responsible for the collection and handling of the samples, evaluating the immunohistochemical stainings, making the statistical work, and writing the article

Päivi Heikkilä: planning the study and writing the article, analyzing the stainings.

Funding This work was supported by the Helsinki University Central Hospital Research Foundation, the Finnish Cancer Foundation, the Sigrid Juselius Foundation, and the Finnish Breast Cancer Group.

\section{Compliance with ethical standards}

The Ethics Committee of the Helsinki University Central Hospital approved the study protocol.

Conflict of interest The authors declare that they have no conflict of interest.

\section{References}

1. Gouon-Evans V, Rothenbeg ME, Pollard JW (2000) Postnatal mammary gland development requires macrophages and eosinophils. Development 127:2269-2282

2. Pollard JW (2009) Trophic macrophages in development and disease. Nat Rev Immunol 9:259-270

3. Mantovani A, Sozzani S, Locati M, Allavena P, Sica A (2002) Macrophage polarization: tumor-associated macrophages as a paradigm for polarized M2 mononuclear phagocytes. Trends Immunol 23:549-555

4. Pollard JW (2004) Tumor-educated macrophages promote tumor progression and metastasis. Nat Rev Cancer 4:71-78

5. Mantovani A, Sica A, Sozzani S, Allavena P, Vecchi A, Locati M (2004) The chemokine system in diverse forms of macrophage activation and polarization. Trends Immunol 25:677-686

6. Zawada AM, Rogacev KS, Rotter B, Winter P, Marell RR, Fliser D, Heine GH (2011) SuperSAGE evidence for CD14++CD16+ monocytes as a third monocyte subset. Blood 118(12):e50-e61

7. Mosser DM, Edwards JP (2008) Exploring the full spectrum of macrophage activation. Nat Rev Immunol 8:958-969
8. Ambarus CA, Krausz S, van Eijk M, Hamann J, Radstake TRDJ, Reedquist KA, Tak PP, Baeten DLP (2012) Systematic validation of specific phenotypic markers for in vitro polarized human macrophages. J Immunol Methods 375:196-206

9. Solinas G, Germano G, Mantovani A, Allavena P (2009) Tumorassociated macrophages (TAM) as major players of the cancerrelated inflammation. J Leukoc Biol 86(5):1065-1073

10. Pollard JW (2008) Macrophages define the invasive microenvironment in breast cancer. J Leukoc Biol 84:623-630

11. Qian B, Li J, Zhang H et al (2011) CCL2 recruits inflammatory monocytes to facilitate breast-tumour metastasis. Nature 475:222225

12. Movahedi K, Laoui D, Gysemans C, Baeten M, Stange G, van den Bossche J, Mack M, Pipeleers D, in't Veld P, de Baetselier P, van Ginderachter JA (2010) Different tumor microenvironments contain functionally distinct subsets of macrophages derived from Ly6C(high) monocytes. Cancer Res 70:5728-5739

13. Lu X, Kang Y (2009) Chemokine (C-C motif) ligand 2 engages CCR2 + stromal cells of monocytic origin to promote breast cancer metastasis to lung and bone. J Biol Chem 284:29087-29096

14. Li M, Knight DA, Snyder LA, Smyth MJ, Stewart TJ (2013) A role for CCL2 in both tumor progression and immunosurveillance. OncoImmunology 2(7):e25474

15. Zhu X, Fujita L, Snyder A, Okada H (2011) Systemic delivery of neutralizing antibody targeting CCL2 for glioma therapy. J NeuroOncol 104:83-92

16. Hussein MR, Hassan HI (2006) Analysis of the mononuclear inflammatory cell infiltrate in the normal breast, benign proliferative breast disease, in situ and infiltrating ductal breast carcinomas: preliminary observations. J Clin Pathol 59:972-977

17. Volodko N, Reiner A, Rudas M, Jakesz R (1998) Tumourassociated macrophages in breast cancer and their prognostic correlations. Breast 7:99-105

18. Medrek C, Ponten F, Jirstrom K, Leandersson K (2012) The presence of tumor associated macrophages in tumor stroma as a prognostic marker for breast cancer patients. BMC Cancer 12:306-315

19. Leek RD, Lewis CE, Whitehouse R, Greenall M, Clarke J, Harris AL (1996) Association of macrophage infiltration with angiogenesis and prognosis in invasive breast carcinoma. Cancer Res 56: 4625-4629

20. Joensuu K, Leidenius M, Kero M, Andersson LC, Horwitz KB, Heikkilä P (2013) ER, PR, HER2, Ki67 and CK5 in early and late relapsing breast cancer - reduced CK5 expression in metastases. Breast Cancer: Bacic Clin Res 7:23-34

21. Laoui D, Movahedi K, van Overmeire E, van den Bossche J, Schouppe E, Mommer C, Nikolaou A, Morias Y, de Baetselier P, van Ginderachter JA (2011) Tumor-associated macrophages in breast cancer: distinct subsets, distinct functions. Int J Dev Biol 55:861-867

22. Yang J, Zhang L, Yu C, Yang X-F, Wang H (2014) Monocyte and macrophage differentiation: circulation inflammatory monocyte as biomarker for inflammatory diseases. Biomarker Research 2:1-9

23. Loberg RD, Ying C, Craig M, Day LL, Sargent E, Neeley C, Wojno K, Snyder LA, Yan L, Pienta KJ (2007) Targeting CCL2 with systemic delivery of neutralizing antibodies induces prostate cancer tumor regression in vivo. Cancer Res 67:9417-9424

24. Conti I, Rollins BJ (2004) CCL2 (monocyte chemoattractant protein-1) and cancer. Semin Cancer Biol 14:149-154

25. Roca H, Varsos ZS, Sud S, Craig MJ, Ying C, Pienta KJ (2009) CCL2 and Interleukin-6 promote survival of human CD11b + peripheral blood mononuclear cells and induce M2-type macrophage polarization. J Biol Chem 284(49):34342-34354

26. Querzoli P, Albonico G, Ferretti S, Rnaldi R, Magri E, Indelli M, Nenci I (1996) MIB-1 proliferative activity in invasive breast cancer measured by image analysis. J Clin Pathol 49(11):926-930 
27. Taneja P, Maglic KF, Zhu S, Kendig RD, Fry EA, Inoue K (2010) Classical and novel prognostic markers for breast cancer and their clinical significance. Clin Med Insights Oncol 4:15-34

28. Allavena P, Signorelli M, Chiappa M et al (2005) Antiinflammatory properties of the novel antitumor agent yondelis (trabectedin): inhibition of macrophage differentiation and cytokine production. Cancer Res 65:2964-2971

29. Frow EK, Reckless J, Grainger DJ (1999) Tools for antiinflammatory drug design: in vitro models of leukocyte migration. Med Res Rev 24(3):276-282

30. Nywening TM, Wang-Gillam A, Sanford DA et al (2016) Phase 1b study targeting tumour associated macrophages with CCR2 inhibition plus FOLFIRINOX in locally advanced and borderline resectable pancreatic cancer. Lancet Oncol 17(5):651-662. https:// doi.org/10.1016/S1470-2045(16)00078-4

31. Giraudo E, Inoue M, Hanahan D (2004) An amino-bisphosphonate targets MMP-9-expressing macrophages and angiogenesis to impair cervical carcinogenesis. J Clin Invest 114:623-633

32. Kitamura T, Qian B-Z, Soong D, Cassetta L, Noy R, Sugano G, Kato Y, Li J, Pollard JW (2015) CCL2-induced chemokine cascade promotes breast cancer metastasis by enhancing retention of metastasis-associated macrophages. J Exp Med 212(7):1043-1059

33. Mathenge E, Dean C, Clements D et al (2014) Core needle biopsy of breast Cancer tumors increases distant metastases in a mouse model. Neoplasia 16:950-960 